\title{
Mortalidade por Câncer de Fígado e Vias Biliares no Brasil: Tendências e Projeções até 2030
}

doi: https://doi.org/10.32635/2176-9745.RBC.2019v65n4.435

\author{
Mortality due to Malignant Neoplasms of the Liver and Bile Ducts in Brazil: Trends and Projections until 2030 \\ Mortalidad por Cáncer de Hígado y Biliar en Brasil: Tendencias y Proyecciones hasta 2030
}

\begin{abstract}
Flávia Arichelle Cavalcante dos Santos'; Fábia Cheyenne Gomes de Morais Fernandes²; Emelynne Gabrielly de Oliveira Santos; Nayre Beatriz Martiniano Medeiros ${ }^{4}$; Dyego Leandro Bezerra de Souza5; Isabelle Ribeiro Barbosa ${ }^{6}$
\end{abstract}

\section{Resumo}

Introduçáo: A neoplasia de fígado e vias biliares intra-hepáticas é a sétima mais incidente e representa a segunda maior causa de morte por câncer no mundo. Sendo assim, é crucial compreender a epidemiologia dessa doença, no que diz respeito às tendências temporais da mortalidade e da carga que essa doença apresentará no futuro. Objetivo: Analisar a tendência da mortalidade por câncer de fígado e vias biliares no Brasil e calcular as projeçóes de mortalidade até 2030. Método: Estudo ecológico baseado em óbitos por neoplasia maligna de fígado e vias biliares intra-hepáticas (C22) ocorridos no Brasil no período de 2001 a 2015 e registrados no Sistema de Informação sobre Mortalidade. As tendências de mortalidade foram analisadas pela regressão Joinpoint; para o cálculo das projeçôes, foi utilizado o programa Nordpred. Resultados: Para o sexo feminino, houve redução das taxas de mortalidade nas Regióes Centro-Oeste, Sudeste e Norte no Brasil; para o sexo masculino, essas Regiôes apresentaram tendências de aumento, porém não significativo. As taxas de mortalidade para o sexo feminino apresentarão reduçóes no futuro, com destaque para as taxas das Regiōes Norte e Nordeste, com redução de cerca de $30 \%$ até 2030. Para o sexo masculino, haverá acréscimo de 12\% nas taxas de mortalidade para a Região Sul. Conclusáo: A mortalidade por câncer de fígado e vias biliares no Brasil apresenta tendência de redução para o sexo feminino e estabilidade para o sexo masculino, e essa característica será mantida nas próximas décadas.

Palavras-chave: Neoplasias Hepáticas/mortalidade; Neoplasias Hepáticas/epidemiologia; Neoplasias dos Ductos Biliares/mortalidade; Neoplasias dos Ductos Biliares/epidemiologia; Previsóes.

\begin{abstract}
Introduction: Intrahepatic liver and biliary tract neoplasm is the seventh most incident and represents the second leading cause of cancer death in the world. Therefore, it is crucial to understand the epidemiology of this disease in relation to the temporal trends of mortality and burden that this disease will present in the future. Objective: To analyze the trend in mortality by liver and biliary cancer in Brazil and to calculate mortality projections until 2030. Method: An ecological study based in deaths from malignant liver and bile duct cancer (C22) occurred in Brazil from 2001 to 2015 and recorded in the Mortality Information System. Mortality trends were analyzed by Joinpoint regression, while for the calculation of projections, the Nordpred program was used. Results: For females, there was a reduction in mortality rates in the Midwest, Southeast and North regions in Brazil; for males, these regions showed increasing but no significant trends. Mortality rates for females will decrease in the future, with emphasis for the rates in the North and Northeast, with a reduction of about $30 \%$ by 2030 . For males, there will be a $12 \%$ increase in mortality rates for the Southern Region. Conclusion: Mortality due to liver and biliary cancer in Brazil shows a tendency of reduction for females and stability for males, and this characteristic will be maintained in the coming decades.

Key words: Liver Neoplasms/mortality; Liver Neoplasms/epidemiology; Bile Duct Neoplasms/mortality; Bile Duct Neoplasms/epidemiology; Forecasting.
\end{abstract}

Resumen

Introducción: La neoplasia intrahepática del hígado y del tracto biliar es el séptimo cáncer más común y representa la segunda causa principal de muerte por cáncer en el mundo. Por lo tanto, es crucial comprender la epidemiología de esta enfermedad con respecto a las tendencias temporales de mortalidad y carga que esta enfermedad presentará en el futuro. Objetivo: Analizar la tendencia de la mortalidad por cáncer de hígado y biliar en Brasil y calcular las proyecciones de mortalidad para 2030. Método: Un estudio ecológico basado en las muertes por cáncer de hígado y conducto biliar (C22) en Brasil en el de 2001 a 2015 y registrado en el Sistema de Información de Mortalidad. Las tendencias de mortalidad se analizaron mediante regresión de Joinpoint, mientras que para el cálculo de las proyecciones se utilizó el programa Nordpred. Resultados: Para las mujeres, hubo una reducción en las tasas de mortalidad en las regiones del Medio Oeste, Sudeste y Norte no Brasil; para los hombres, estas regiones mostraron tendencias crecientes, pero no significativas. Las tasas de mortalidad para las mujeres disminuirán en el futuro, con énfasis en las tasas en el norte y el noreste, con una reducción de alrededor del $30 \%$ para 2030 . Para los hombres, habrá un aumento del $12 \%$ en las tasas de mortalidad para las mujeres. Conclusión: La mortalidad por cáncer de hígado y biliar en Brasil muestra una tendencia a la reducción de las mujeres y la estabilidad de los hombres, y esta característica se mantendrá en las próximas décadas.

Palabras clave: Neoplasias Hepáticas/mortalidad; Neoplasias Hepáticas/ epidemiología; Neoplasias de los Conductos Biliares/mortalidad; Neoplasias de los Conductos Biliares/epidemiología; Predicción.

\footnotetext{
${ }^{1}$ Faculdade de Ciências da Saúde do Trairi (Facisa). Universidade Federal do Rio Grande do Norte (UFRN). Natal (RN), Brasil. Orcid iD: https://orcid.org/0000-0003-4186-5265 2 UFRN. Natal (RN), Brasil. Orcid iD: https://orcid.org/0000-0002-0834-855X

${ }^{3}$ UFRN. Natal (RN), Brasil. Orcid iD: https://orcid.org/0000-0002-3890-4879

${ }^{4}$ Centro Universitário do Rio Grande do Norte (UNI-RN). Natal (RN), Brasil. Orcid iD: https://orcid.org/0000-0002-8126-239X

${ }^{5}$ UFRN. Natal (RN), Brasil. Orcid id: https://orcid.org/0000-0001-8426-3120

${ }^{6}$ Facisa/UFRN. Natal (RN), Brasil. Orcid iD: https://orcid.org/000-0002-1385-2849

Endereço para correspondência: Emelynne Gabrielly de Oliveira Santos. Rua Joaquim Inácio, 1375, Tirol. Natal (RN), Brasil. CEP 59022-180. E-mail: nellynha_15@hotmail.com
} 


\section{INTRODUÇÃO}

A neoplasia de fígado e vias biliares intra-hepáticas é a sétima mais incidente e representa a segunda maior causa de morte por câncer no mundo. Em 2018, foram registrados 841 mil casos confirmados da doença e 781 mil óbitos relacionados a ela, o que corresponde a $8,2 \%$ de todas as mortes por neoplasia no mundo, perfazendo uma taxa global de incidência de 9,3 casos/100 mil habitantes ${ }^{1}$.

No mundo, as Regiôes mais incidentes da doença estáo entre os países pobres e em desenvolvimento, onde ocorrem cerca de $90 \%$ dos casos, com destaque para a Ásia e a África Subsaariana. Entre os homens, as maiores taxas são encontradas na Ásia Oriental e do Sudeste (31,9 e 22,2 casos/100 mil habitantes, respectivamente); enquanto, nas mulheres, as Regiōes mais incidentes estão na Ásia Oriental e África Ocidental (10,2 e 8,1 casos/100 mil habitantes respectivamente $)^{2-4}$. No Brasil, foram registrados 139.530 mil óbitos por câncer de fígado entre os anos de 1980 e 2010 , com cerca de $60 \%$ de casos entre homens e $40 \%$ em mulheres ${ }^{3,5}$.

O carcinoma hepatocelular representa $70 \%$ a $85 \%$ das neoplasias hepáticas primárias e é o tumor primário do fígado mais frequentemente observado. Por outro lado, o colangiocarcinoma, que tem origem nos colangiócitos, células epiteliais que revestem os canais biliares, constituem de $10 \%$ a $15 \%$ das neoplasias hepáticas primárias. Os demais 5\% instituem tumores incomuns, como o angiossarcoma primário hepático, o hemangioendotelioma epitelioide hepático, o hemangiopericitoma, ou o linfoma hepático primário ${ }^{6}$.

De modo geral, apenas a minoria dos casos do carcinoma hepatocelular é passível de intervenção cirúrgica potencialmente curativa. Quando não é realizada intervençáo sobre o tumor, ele costuma crescer progressivamente como uma massa que reduz a função hepática e gera metástases intra e extra-hepáticas. Nesses casos, a morte costuma ocorrer no tempo médio de dez meses. Pacientes com fatores de risco para o carcinoma hepatocelular devem ser submetidos a rastreamento periódico, com custo-efetividade amplamente demonstrado ${ }^{7-9}$.

Os fatores de risco reconhecidos para o hepatocarcinoma compreendem infecção crônica pelo vírus da hepatite $\mathrm{B}$ $(\mathrm{HBV})$ e pelo vírus da hepatite $\mathrm{C}(\mathrm{HCV})$, exposição à aflatoxina na dieta, doença hepática gordurosa, cirrose induzida pelo álcool, obesidade, tabagismo, diabetes e sobrecarga de ferro. Para o colangiocarcinoma, os fatores de risco incluem o derrame de fígado e a cirrose $e^{10,11}$.

Como um número significativo de fatores de risco de câncer hepático é modificável, existe uma forte perspectiva de reduzir sua incidência e mortalidade por meio de estratégias preventivas, como modificação do estilo de vida e imunização contra a hepatite. Alguns de seus fatores etiológicos, incluindo infecção por hepatite e cirrose, são facilmente identificados por exames que podem minimizar o desenvolvimento de câncer de fígado ${ }^{11}$.

Sendo assim, é crucial compreender a epidemiologia dessa doença, no que diz respeito às tendências temporais da mortalidade e da carga que essa doença apresentará no futuro. Além disso, analisar os padróes geográficos e identificar populaçôes de alto risco podem ajudar a compreender a extensão das estratégias preventivas implementadas e oferecer informaçáo para os formuladores de políticas com relação às perspectivas futuras para a prevenção desse câncer no Brasil.

O objetivo deste estudo foi analisar a tendência temporal da mortalidade por câncer de fígado e vias biliar intra-hepáticas e projetar o número de óbitos até 2030 para o Brasil.

\section{MÉTODO}

Estudo ecológico de série temporal, baseado em dados secundários registrados no Sistema de Informação sobre Mortalidade (SIM) do Departamento de Informática do Sistema Único de Saúde (DATASUS). Foram analisados os óbitos decorrentes da neoplasia maligna de fígado e vias biliares intra-hepáticas (C22) categorizadas a partir da Classificação Estatística Internacional de Doenças e Problemas Relacionados à Saúde - 10a Revisão (CID-10), ocorridos no Brasil no período de 2001 a 2015 e analisados de acordo com o sexo, faixa etária e as Regióes brasileiras.

Apesar de, nos últimos anos, reconhecer-se que o SIM no Brasil obteve um ganho significativo de qualidade, a utilizaçâo de dados secundários sobre mortalidade está sujeita a sub-registro. Para correçấo do sub-registro de óbitos por neoplasia maligna de Fígado e vias biliares intra-hepáticas, foram utilizadas informaçóes da Redistribuição por Capítulos dos Óbitos corrigidos pela Pesquisa de Busca Ativa, iniciativa realizada pelo Ministério da Saúde, com dados disponibilizados no sítio do DATASUS ${ }^{12}$.

Foi calculado o fator de correção para cada faixa etária $^{13}$, período, Regiāo e sexo, a partir da diferença em percentual entre a quantidade de óbitos notificados ao SIM e a de óbitos redistribuídos, baseado no Capítulo II (Neoplasias) da CID-10. Tal diferença foi expressa em valores decimais com o valor 1 correspondendo a uma mudança de $100 \%$, por exemplo, existindo a possibilidade de valores maiores, visto que, em algumas localidades, o valor redistribuído representou valores acima daqueles registrados no SIM. Além disso, casos em que o valor redistribuído foi inferior aos registrados no SIM apresentaram uma diferença negativa. 
ONDE: $\mathrm{D}$ = diferença entre óbitos redistribuídos e óbitos registrados no SIM por Neoplasias em relação ao número de óbitos registrados no SIM por Neoplasias; NR = número de óbitos redistribuídos por Neoplasias; NS = número de óbitos registrados no SIM por Neoplasias.

Essa diferença obtida foi somada ao valor 1 para calcular o fator de correçáo, visto que o número 1 representa o fator neutro em uma multiplicação, de acordo com a fórmula a seguir:

$$
F=1+D
$$

ONDE: $\mathrm{F}$ = fator de correção do capítulo II (Neoplasias); $\mathrm{D}$ = diferença entre óbitos redistribuídos e óbitos registrados no SIM por Neoplasias, em relação ao número de óbitos registrados no SIM por Neoplasias.

Esse fator foi multiplicado ao número de óbitos por câncer. Assim, foi pressuposto que o fator de correção para o Capítulo II poderia ser aplicável ao câncer de fígado e vias biliares intra-hepáticas. A fórmula usada para esse cálculo está descrita a seguir:

$$
O C=F \times N O S
$$

ONDE: $\mathrm{OC}=$ número de óbitos por neoplasia maligna de fígado e vias biliares intra-hepáticas corrigidos; NOS = número de óbitos registrados no SIM por neoplasia maligna de fígado e vias biliares intra-hepáticas; $\mathrm{F}$ = fator de correção do capítulo II (neoplasias).

Com a informação do número reajustado de mortes, foram calculadas as taxas de mortalidade padronizadas, ajustadas de acordo com a população mundial por 100 mil habitantes (ASW/100 mil hab.). Os dados de população por Região, por sexo e por idade foram obtidos das informaçôes dos Censos demográficos e das projeçōes intercensitárias, no sítio do Instituto Brasileiro de Geografia e Estatística.

Foi analisada a tendência temporal da mortalidade por câncer de fígado e vias biliares intra-hepáticas no Brasil e Regiōes brasileiras, e calculadas as projeçōes de mortalidade até o ano de 2030 por quinquênio, para os períodos de 2016-2020, 2021-2025 e 2026-2030.

Para analisar as tendências de mortalidade, foi realizada a análise de regressão Joinpoint, utilizando o software Joinpoint Regression Program (National Cancer Institute, Bethesda, Maryland, USA), versão 4.4.0., de janeiro de 2017. O objetivo da análise é identificar a ocorrência de possíveis joinpoints, ponto no qual uma mudança significativa na tendência tenha ocorrido.

O método aplicado identificou joinpoints baseado no modelo com no máximo três pontos de mudança. O modelo final selecionado foi o mais ajustado, com o Annual Percentage Change (APC) baseado na tendência de cada segmento, estimando se esses valores são estatisticamente significativos em um nível de 0,05. Os testes de significância utilizados baseiam-se no método de permutação de Monte Carlo e no cálculo da variação percentual anual da razão, utilizando o logaritmo da razáo ${ }^{14}$.

$\mathrm{Na}$ descrição das tendências, os termos "aumento significativo" ou "redução significativa" indicam que o declive da tendência é estatisticamente significativo $(\mathrm{p}<0,05)$.

As predições foram feitas para cada período utilizando-se o modelo idade-período-coorte do programa Cancer Registry of Norway (Nordpred), Oslo, Norway, inscrito no programa estatístico R. Os dados foram compilados em blocos de cinco anos e o grupo de idade limite considerado para a análise foi o primeiro com mais de dez casos para o período combinado.

Os resultados das prediçóes estâo apresentados no total de óbitos observados e esperados para cada período para o Brasil e as cinco Regióes brasileiras. Para cada período, foram calculadas as taxas de mortalidade ajustadas com base na população padrão mundial para comparaçôes globais, expressas por 100 mil habitantes por ano (ASW/100 mil hab. $)^{15}$. Foram calculadas as mudanças anuais no número de óbitos no último período projetado (2026-2030) comparado ao último período observado (2011-2015), no qual a proporção dessa mudança relaciona-se com as mudanças nos riscos ou demográficas (tamanho ou estrutura da população). Esses dois componentes podem ser diferentes de zero e apresentar uma direção positiva ou negativa. $\mathrm{O}$ cálculo pode ser expresso conforme se segue ${ }^{16}$.

$\Delta t o t=\Delta$ risk $+\Delta p o p=\left(N\right.$ fff $\left.-N_{\text {off }}\right)+\left(N_{\text {off }}-N_{o o o}\right)$

ONDE: $\Delta$ tot é a mudança total, $\Delta$ risk é a mudança em funçáo do risco, $\Delta$ pop é a mudança em função da população, Nooo é o número de casos observados, Nfffé o número de casos projetados, e Noffé o número de casos esperados quando as taxas de mortalidade aumentam durante o período observado.

\section{RESULTADOS}

No período de 2001 a 2015, ocorreram 125.751 óbitos por neoplasia maligna de fígado e vias biliares intra-hepáticas no Brasil, com 56,9\% afetando o sexo masculino e $43,1 \%$ dos óbitos, o sexo feminino. A taxa de mortalidade padronizada no que diz respeito à populaçáo mundial para as mulheres no Brasil variou de 3,57 óbitos/100 mil habitantes, no ano 2001, a 3,39 óbitos/100 mil habitantes, no ano de 2015. Para os homens, essa taxa variou de 4,23 óbitos/100 mil habitantes, no ano 2001, a 5,84 óbitos/100 mil habitantes, no ano de 2015. A razão entre os sexos para o período de 2001 a 2015 foi, em média, de 1,6. As taxas de mortalidade mais elevadas para ambos os sexos foram registradas na Região Norte, 
com destaque para altas taxas de mortalidade para o sexo feminino na Regiáo Nordeste, e para o sexo masculino no Sul do país (Figura 1).

$\mathrm{Na}$ análise da série histórica das taxas de mortalidade para o sexo feminino, houve uma tendência de aumento significativo da mortalidade para Brasil até o ano de 2005 $(\mathrm{APC}=3,2 \%$ IC95\% 0,6-5,9), seguido de um período de redução, porém sem significância estatística. Destaca-se ainda a redução apresentada pelas Regiōes Centro-Oeste $(\mathrm{APC}=-2,0 \% \mathrm{IC} 95 \%-3,1 ;-0,8)$, Sudeste $(\mathrm{APC}=-1,0 \%$
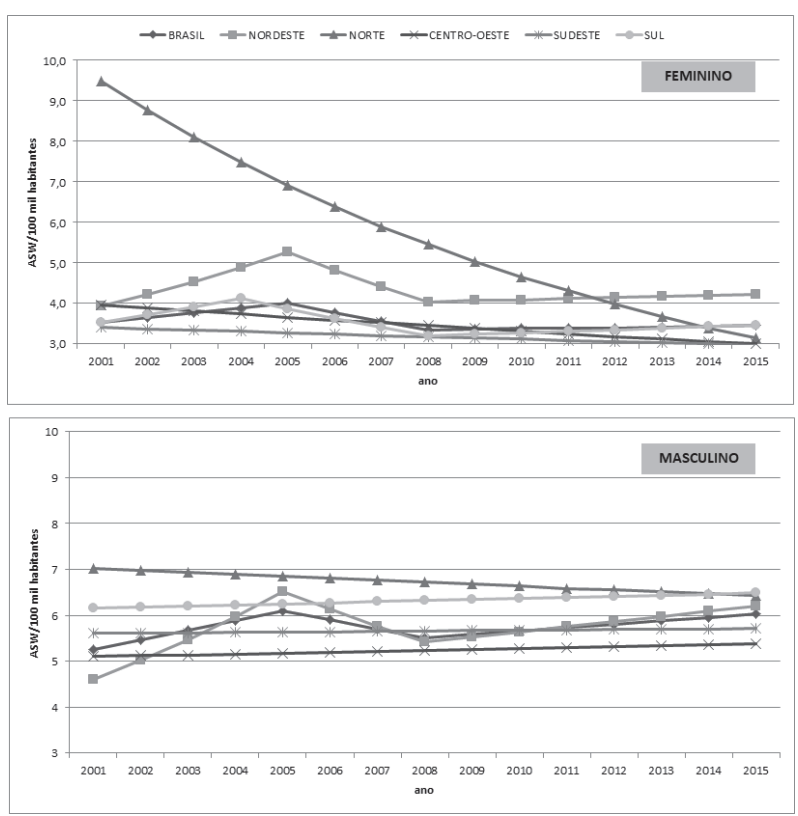

Figura 1. Taxas padronizadas de mortalidade do câncer de fígado e vias biliares para o Brasil e Regiões brasileiras, de acordo com o sexo, para o período 2001-2015

Legenda: Taxas de mortalidade ajustadas à populaçăo mundial (ASW/100 mil hab.).
IC95\% - 1,7; -0,3) e Norte (APC = -7,6\% IC95\% - 12,7; -2,2). A mortalidade masculina apresentou tendência de aumento no Brasil (APC = 3,8\% IC95\% 0,9-6,8) até o ano de 2005, seguido por um período de redução, porém não significativa. O mesmo padrão foi observado para Região Nordeste. Para as demais Regiôes, não houve mudanças significativas (Tabela 1 ).

As Tabelas 2 e 3 apresentam o número de óbitos e as taxas de mortalidade padronizadas para os períodos observados e projetados, para o sexo feminino e masculino, respectivamente. Ao analisar os dados para o Brasil no quinquênio 2026-2030, foi projetada a ocorrência de 34.028 óbitos femininos por câncer de fígado; enquanto, para o sexo masculino, esse número foi de 48.819 óbitos. As taxas de mortalidade para o sexo feminino apresentarão redução acentuada ao longo do período de 2016 a 2030, com destaque para as taxas das Regióes Norte e Nordeste, com redução de cerca de $30 \%$ quando comparado ao período de 2001 a 2005. Para o sexo masculino, observa-se outro padrão, com elevação das taxas de mortalidade até 2030, com maior aumento para a Regiáo Sul. Quanto à faixa etária, o maior número de mortes se concentra na faixa acima de 60 anos de idade.

$\mathrm{Na}$ Figura 2, são apresentadas as taxas de mortalidade por câncer de fígado, nos períodos observados e projetados, de acordo com a influência dos riscos e da estrutura populacional do Brasil e Regiōes. Para ambos os sexos, verifica-se que as diferenças nas taxas, que serão observadas até 2030, serão explicadas pela mudança na estrutura demográfica brasileira. Destaca-se a reduçáo das mortes relacionadas aos fatores de risco da doença na maioria das Regiōes brasileiras para ambos os sexos, ressaltando o Norte e o Centro-Oeste.

Tabela 1. Tendências da mortalidade por câncer de fígado e vias biliares no Brasil e Regiões: número de óbitos, annual percentage change, intervalo de confiança (IC 95\%) e ano do joinpoint

\begin{tabular}{lcccc}
\hline & Número de mortes & APC1 $($ IC 95\%) & Joinpoint & APC2 (IC 95\%) \\
\hline FEMININO & & & & \\
Brasil & 54.308 & $3,2^{*}(0,6 ; 5,9)$ & 2005 & $-5,9(-13,1 ; 2)$ \\
Nordeste & 17.210 & $7,5^{*}(2,0 ; 13,4)$ & 2005 & $-8,4(-22,6 ; 8,4)$ \\
Norte & 3.375 & $-7,6^{*}(-12,7 ;-2,2)$ & & \\
Centro-Oeste & 2.968 & $-2,0^{*}(-3,1 ;-0,8)$ & & \\
Sudeste & 23.012 & $-1,0^{*}(-1,7 ;-0,3)$ & & \\
Sul & 8.636 & $5,1(-2,6 ; 13,5)$ & 2004 & $-6,1(-13,0 ; 1,3)$ \\
MASCULINO & & & & $-3,3(-11,6 ; 5,7)$ \\
Brasil & 71.443 & $3,8^{*}(0,9 ; 6,8)$ & 2005 & $-5,9(-21,4 ; 12,6)$ \\
Nordeste & 18.583 & $9,1^{*}(3,1 ; 15,5)$ & 2005 & \\
Norte & 5.145 & $-0,6(-1,6 ; 0,3)$ & & \\
Centro-Oeste & 4.284 & $0,4(-1,1 ; 1,9)$ & & \\
Sudeste & 31.967 & $0,1(-0,4 ; 0,6)$ & & \\
Sul & 12.522 & $0,4(-0,5 ; 1,2)$ & & \\
\hline
\end{tabular}

Legenda: $\mathrm{APC}=$ Annual percentage change; ${ }^{*}$ Significância estatística p $<0,05$. 
Tabela 2. Mortalidade por câncer de fígado e vias biliares no Brasil e Regiões em para o sexo feminino: número de óbitos observados e projetados por idade e taxas de mortalidade ajustadas à população mundial

\begin{tabular}{|c|c|c|c|c|c|c|}
\hline & \multicolumn{3}{|c|}{ Período observado } & \multicolumn{3}{|c|}{ Período projetado } \\
\hline & 2001-2005 & 2006-2010 & 2011-2015 & 2016-2020 & 2021-2025 & 2016-2030 \\
\hline \multicolumn{7}{|l|}{ BRASIL } \\
\hline \multicolumn{7}{|c|}{ Idade (anos) } \\
\hline $0-39$ & 812 & 738 & 789 & 774 & 728 & 723 \\
\hline $40-59$ & 3.856 & 4.310 & 4.884 & 5.355 & 5.655 & 5.998 \\
\hline $60-85$ & 10.923 & 12.711 & 15.293 & 18.640 & 22.694 & 27.307 \\
\hline ASW & 3,57 & 3,42 & 3,39 & 3,36 & 3,33 & 3,29 \\
\hline \multicolumn{7}{|c|}{ NORDESTE } \\
\hline \multicolumn{7}{|c|}{ Idade (anos) } \\
\hline $0-39$ & 282 & 273 & 274 & 277 & 265 & 252 \\
\hline $40-59$ & 1.352 & 1.324 & 1.480 & 1.540 & 1.615 & 1.731 \\
\hline $60-85$ & 3.498 & 4.014 & 4.703 & 5.537 & 6.456 & 7.468 \\
\hline ASW & 4,45 & 4,28 & 4,17 & 4,02 & 3,86 & 3,7 \\
\hline \multicolumn{7}{|l|}{ NORTE } \\
\hline \multicolumn{7}{|c|}{ Idade (anos) } \\
\hline $0-39$ & 79 & 87 & 77 & 78 & 76 & 74 \\
\hline $40-59$ & 300 & 316 & 340 & 363 & 404 & 469 \\
\hline $60-85$ & 628 & 703 & 845 & 994 & 1.175 & 1.376 \\
\hline ASW & 4,89 & 4,44 & 4,11 & 3,74 & 3,46 & 3,25 \\
\hline \multicolumn{7}{|c|}{ CENTRO-OESTE } \\
\hline \multicolumn{7}{|c|}{ Idade (anos) } \\
\hline $0-39$ & 40 & 59 & 48 & 41 & 39 & 37 \\
\hline $40-59$ & 184 & 272 & 326 & 327 & 333 & 332 \\
\hline $60-85$ & 425 & 673 & 777 & 950 & 1.149 & 1.382 \\
\hline ASW & 2,8 & 3,4 & 3,08 & 2,76 & 2,53 & 2,35 \\
\hline \multicolumn{7}{|c|}{ SUDESTE } \\
\hline \multicolumn{7}{|c|}{ Idade (anos) } \\
\hline $0-39$ & 303 & 247 & 297 & 267 & 254 & 247 \\
\hline $40-59$ & 1.552 & 1.828 & 2.018 & 2.202 & 2.280 & 2.375 \\
\hline $60-85$ & 4.761 & 5.457 & 6.558 & 7.885 & 9.556 & 11.503 \\
\hline ASW & 3,2 & 3,05 & 3,02 & 2,98 & 2,97 & 2,96 \\
\hline \multicolumn{7}{|l|}{ SUL } \\
\hline \multicolumn{7}{|c|}{ Idade (anos) } \\
\hline $0-39$ & 98 & 80 & 95 & 104 & 106 & 106 \\
\hline $40-59$ & 550 & 614 & 756 & 879 & 964 & 1.049 \\
\hline $60-85$ & 1.869 & 2.034 & 2.531 & 3.229 & 4.126 & 5.161 \\
\hline ASW & 3,59 & 3,23 & 3,35 & 3,52 & 3,69 & 3,84 \\
\hline
\end{tabular}

Legenda: Taxas de mortalidade ajustadas à populaçáo mundial (ASW/100 mil hab.).

\section{DISCUSSÃO}

Os resultados do presente estudo apontam que há uma tendência de redução da mortalidade por câncer de fígado e vias biliares para o sexo feminino na maioria das Regiōes brasileiras e, até 2030, essas taxas continuarão a reduzir. Já para o sexo masculino, a tendência é de aumento não significativo da mortalidade, embora essa população represente as mais elevadas taxas.

As taxas de mortalidade por câncer de fígado e vias biliares apresentam importantes variações regionais. No presente estudo, as taxas de mortalidade para o sexo masculino se assemelharam às encontradas na Ásia Central e do Sul (3,6 óbitos por 100 mil hab.), no Norte da Europa (4,0 óbitos por 100 mil hab.) e na África Oriental (4,6 óbitos por 100 mil hab.). Para o sexo feminino, as taxas brasileiras foram superiores às do Norte da Europa $(1,8$ óbitos por 100 mil hab.), da Austrália/Nova Zelândia (2,0 óbitos por 100 mil hab.), da Europa Ocidental (2,1 óbitos por 100 mil hab.) e do Centro-Sul da Ásia (2,1 óbitos por 100 mil hab.), para os quais foram relatadas as menores taxas de mortalidade ${ }^{11}$. 
Tabela 3. Mortalidade por câncer de fígado e vias biliares no Brasil e Regiões para o sexo masculino: número de óbitos observados e projetados por idade e taxas de mortalidade ajustadas à população mundial

\begin{tabular}{|c|c|c|c|c|c|c|}
\hline & \multicolumn{3}{|c|}{ Período observado } & \multicolumn{3}{|c|}{ Período projetado } \\
\hline & 2001-2005 & 2006-2010 & 2011-2015 & 2016-2020 & 2021-2025 & 2016-2030 \\
\hline \multicolumn{7}{|l|}{ BRASIL } \\
\hline \multicolumn{7}{|c|}{ Idade (anos) } \\
\hline $0-39$ & 1.003 & 1.225 & 1.206 & 1.008 & 834 & 681 \\
\hline $40-59$ & 4.538 & 7.358 & 8.156 & 8.575 & 8.574 & 8.552 \\
\hline $60-85$ & 9.495 & 15.044 & 19.277 & 25.173 & 32.102 & 39.585 \\
\hline ASW & 4,23 & 5,69 & 5,84 & 5,97 & 5,97 & 5,86 \\
\hline \multicolumn{7}{|c|}{ NORDESTE } \\
\hline \multicolumn{7}{|c|}{ Idade (anos) } \\
\hline $0-39$ & 442 & 429 & 422 & 404 & 376 & 352 \\
\hline $40-59$ & 1.448 & 1.744 & 1.918 & 2.113 & 2.253 & 2.396 \\
\hline $60-85$ & 3.319 & 3.964 & 4.885 & 6.022 & 7.315 & 8.711 \\
\hline ASW & 5,46 & 5,76 & 5,98 & 6,13 & 6,12 & 5,99 \\
\hline \multicolumn{7}{|l|}{ NORTE } \\
\hline \multicolumn{7}{|c|}{ Idade (anos) } \\
\hline $0-39$ & 187 & 215 & 218 & 236 & 244 & 246 \\
\hline $40-59$ & 486 & 548 & 602 & 640 & 700,8 & 804 \\
\hline $60-85$ & 798 & 933 & 1.158 & 1.408 & 1.707 & 2.029 \\
\hline ASW & 6,93 & 6,76 & 6,6 & 6,32 & 6,07 & 5,84 \\
\hline \multirow{2}{*}{\multicolumn{7}{|c|}{$\begin{array}{c}\text { CENTRO-OESTE } \\
\text { Idade (anos) }\end{array}$}} \\
\hline & & & & & & \\
\hline $0-39$ & 90 & 98 & 75 & 78 & 79 & 79 \\
\hline $40-59$ & 370 & 452 & 558 & 596 & 591 & 579 \\
\hline $60-85$ & 648 & 876 & 1.112 & 1.455 & 1.889 & 2.369 \\
\hline ASW & 4,94 & 5,23 & 5,26 & 5,26 & 5,19 & 5,05 \\
\hline \multicolumn{7}{|c|}{ SUDESTE } \\
\hline \multicolumn{7}{|c|}{ Idade (anos) } \\
\hline $0-39$ & 461 & 426 & 415 & 411 & 400 & 402 \\
\hline $40-59$ & 2.718 & 3.366 & 3.618 & 3.831 & 3.810 & 3.817 \\
\hline $60-85$ & 5.550 & 6.688 & 8.738 & 11.094 & 13.931 & 16.849 \\
\hline ASW & 5,42 & 5,5 & 5,63 & 5,67 & 5,63 & 5,5 \\
\hline \multicolumn{7}{|c|}{ SUL } \\
\hline \multicolumn{7}{|c|}{ Idade (anos) } \\
\hline $0-39$ & 105 & 111 & 120 & 123 & 122 & 120 \\
\hline $40-59$ & 984 & 1.332 & 1.516 & 1.642 & 1.686 & 1.733 \\
\hline $60-85$ & 2.173 & 2.716 & 3.481 & 4.488 & 5.722 & 7.023 \\
\hline ASW & 5,84 & 6,21 & 6,35 & 6,51 & 6,62 & 6,65 \\
\hline
\end{tabular}

Legenda: Taxas de mortalidade ajustadas à população mundial (ASW/100 mil hab.).

Em relação às tendências da mortalidade brasileira observadas no presente estudo, esses achados são confirmados pelos resultados da pesquisa que utilizou dados dos Registros de câncer de Base Populacional dos países da América latina, para o período de 1997 a 2006. Nesta pesquisa, foi observada, para o sexo masculino, uma tendência de redução não significativa das taxas de mortalidade para a Argentina (APC $=-1,2 \%$ ), Chile $(\mathrm{APC}=-1,8 \%)$ e Costa Rica $(\mathrm{APC}=-1,7 \%)$ e verificado um aumento não significativo para o Brasil $(\mathrm{APC}=$ $2,0 \%)$. Esse padrão foi semelhante para o sexo feminino, com redução não significativa para a Argentina (APC $=-2,4 \%)$, Chile $(\mathrm{APC}=-0,3 \%)$ e Costa Rica $(\mathrm{APC}=$ $-3,5 \%)$, e tendência de aumento não significativo para o Brasil $(\mathrm{APC}=0,9 \%)^{17}$. Confirmando os achados dessa pesquisa, o estudo sobre a mortalidade por cirrose, câncer hepático e transtornos devidos ao uso de álcool, utilizando dados sobre a Carga Global de Doenças no Brasil, mostrou que houve tendência de estabilidade da mortalidade por câncer hepático no Brasil no período de 1990 a $2015^{10}$.

A tendência de estabilidade ou de reduçáo da mortalidade por câncer de fígado e vias biliares não é um 


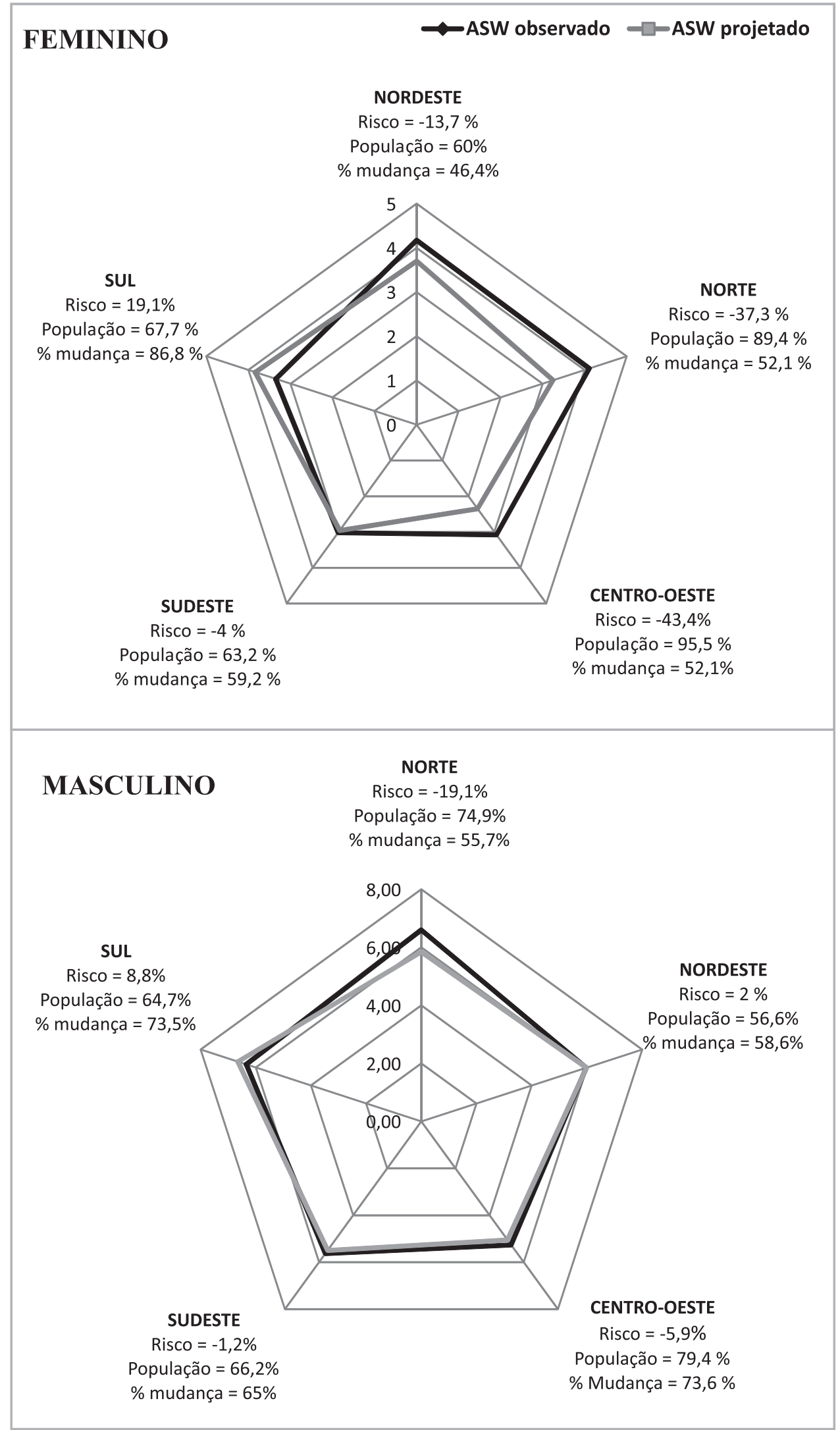

Figura 2. Taxas padronizadas pela população mundial, mudança total (mudança), mudança relativa em razão dos fatores de risco (risco) e mudança relativa à estrutura da população (população), para ambos os sexos, entre 201 1-2015 (observado) e $2026-2030$ (projetado) da mortalidade por câncer de fígado e vias biliares no Brasil

Legenda: Taxas de mortalidade ajustadas à populaçáo mundial (ASW/100 mil hab.). 
padrão nos continentes europeu, asiático e americano. Utilizando dados do Globocan para 184 países no ano de 2012, o estudo de Wong et al. ${ }^{11}$ mostrou que há uma tendência de aumento da mortalidade por câncer de fígado e vias biliares para os Estados Unidos (Average Annual Percent Change - AAPC $=3,1 \%$, IC95\% $=2,7$ 3,4 em homens; AAPC $=2,3 \%$, IC95\% 1,1-3,5 em mulheres) e no Canadá (AAPC $=2,2 \%$ IC95\% $=1,5$ $3,0 \mathrm{em}$ homens; $\mathrm{AAPC}=1,9 \%$ IC95\% $\%=0,3-3,6 \mathrm{em}$ mulheres); na Noruega (AAPC $=5,6 \%$ IC95\% $=2,8$ 8,5 ), Reino Unido (AAPC $=4,7 \%$ IC95\% $=3,6-5,8$ ), Lituânia (AAPC $=2,5 \%$ IC95\% = 0,8-4,3) e Holanda $(\mathrm{AAPC}=2,0 \%, \mathrm{IC} 95 \%=0,7-3,3)$ apresentaram aumento da mortalidade em homens. $\mathrm{Na}$ Singapura (AAPC $=7,7$, IC95\% $=4,1-11,5)$, houve aumento bastante significativo em mulheres.

As taxas de mortalidade por câncer de fígado e vias biliares apresentadas por esses países são explicadas, entre outros fatores, pelas variaçóes nas taxas de incidência por esse câncer. Em um estudo que analisou a base de dados do Cancer Incidence in Five Continents (CI5plus), foram observados distintos padrôes de incidência ao se comparar Europa, Ásia e Américas para o período de 2003 a 2007. Países como Japão (APC $=-4,9 \%)$, China $(\mathrm{APC}=$ $-1,7 \%)$ e Singapura $(\mathrm{APC}=-2,0 \%)$ apresentaram redução significativa das taxas; Brasil (APC $=3,7 \%)$ e Colômbia $(\mathrm{APC}=2,8 \%)$ apresentaram aumento não significativo, enquanto Holanda $(\mathrm{APC}=1,9 \%)$, Alemanha $(\mathrm{APC}=$ $3,3 \%)$ e Suíça $(\mathrm{APC}=1,4 \%)$ apresentaram tendência de aumento da incidência ${ }^{2}$.

A epidemiologia desse câncer está diretamente relacionada à prevalência dos fatores de risco já descritos, que também ocorrem de forma desigual nas macrorregiôes brasileiras. Entre as exposiçóes de maior importância, destacam-se a ocorrência das infecçóes pelos $\mathrm{HBV}, \mathrm{HCV}$, o consumo de álcool, a cirrose hepática e a contaminação de alimentos com aflatoxinas ${ }^{6,3,10,11}$. A análise da prevalência desses fatores nas Regióes do Brasil pode explicar as taxas de mortalidade por câncer hepático serem mais elevadas nas no Norte, Nordeste e Sul do país, bem como na tendência apresentada por essas Regiōes nas últimas décadas.

No final do século 20, o Brasil foi classificado como tendo endemicidade moderada por hepatite B. A análise detalhada mostrou uma distribuição altamente heterogênea da doença no Brasil, com a prevalência aumentando do Sul para o Norte do país, sendo a Região Amazônica a de maior endemicidade no Brasil, e as Regióes Sudeste, Nordeste e Centro-Oeste, classificadas como de baixa a moderada prevalência ${ }^{18}$. Em 2010, observou-se que, para populaçáo brasileira de 10 a 69 anos, a soroprevalência global para o anti-HBc total correspondeu a $7,4 \%$, atingindo 10,9\% no grupo das capitais da Regiāo Norte do país. No que se refere ao marcador $\mathrm{HBsAg}$, a prevalência global nas capitais brasileiras foi de $0,37 \%$, atingindo $0,63 \%$ nas capitais do Norte ${ }^{19,20}$. Essas informaçôes são fundamentais, uma vez que as áreas de maior prevalência desse fator de risco, como o Norte e Nordeste do Brasil, são coincidentes com as de maiores taxas de mortalidade apresentadas neste estudo.

No Brasil, o Programa Nacional de Imunização (PNI) tem sido muito exitoso, alcançando uma das mais altas taxas de cobertura de imunização do mundo, e a vacinação contra Hepatite B está entre os programas rotineiros de vacinação universal. Sua cobertura foi ampliada, em 2001, para todo país, como parte do calendário regular para menores de um ano, crianças e adolescentes. A cobertura vacinal das pessoas entre 10 e 19 anos, que haviam recebido ao menos uma dose da vacina, chega a 30\% nas Regiōes Nordeste e Centro-Oeste e Distrito Federal e, para aqueles menores de 1 ano, a cobertura chega a $86,7 \%$. Além disso, o Sistema de Saúde Brasileiro conta com uma política que define o tratamento padrão nos casos de infecção crônica para as hepatites B e C, com todos os medicamentos sendo oferecidos gratuitamente ${ }^{21}$.

Em relação à infecção pelo $\mathrm{HCV}$, estima-se que existam em todo o mundo cerca de 130 a 150 milhóes de infectados pelo $\mathrm{HCV}^{22}$. No Brasil, de três a quatro milhōes de indivíduos são portadores crônicos do vírus. Como náo existe vacina ou imunoglobulina disponível para a prevenção da infecção pelo $\mathrm{HCV}$, o método mais seguro de proteção relaciona-se com atividades de prevenção primária, como triagem e análise de sangue e derivados, de órgãos, esterilização de materiais que podem ser reutilizados, e atividades de prevenção secundária que compreendam a identificação, aconselhamento e testagem de indivíduos em situaçáo de risco; tratamento dos infectados e atividades educativas ${ }^{20,23,24}$.

A dependência e o uso abusivo do álcool também são apontados como uma das principais causas de desenvolvimento da cirrose hepática. Sobre o uso do álcool no Brasil, dados da Pesquisa Nacional de Saúde mostram que $13,7 \%$ dos brasileiros acima de 18 anos fazem uso abusivo dessa substância, sendo mais prevalente nas Regiôes Centro-Oeste (16,2\%) e Nordeste (15,6\%), mais entre os homens $(21,6 \%)$ do que em mulheres $(6,6 \%)^{25}$.

Em um estudo que analisou o uso do álcool como causa necessária da morte, observou-se que as taxas mais elevadas foram registradas para as Regióes Nordeste e Centro-Oeste, e que as taxas de mortalidade ajustadas foram maiores entre os homens em todas as Regiôes, com proporção de risco de oito (Regiōes Norte, Centro-Oeste e Sudeste) a dez vezes maior (Regiáo Sul) para os homens em comparação às mulheres ${ }^{25}$. Fazendo o contraponto com os 
resultados da presente pesquisa, é possível observar que a razão entre os sexos e o aumento dessas taxas na Regiáo Sul do Brasil podem estar relacionados ao comportamento dos homens brasileiros frente ao uso abusivo do álcool. Os danos decorrentes do uso abusivo do álcool vêm diminuindo no Brasil, fato demonstrado na pesquisa, que relatou um declínio na mortalidade por cirrose atribuída ao uso de álcool (de 11,4 para 9,5) $(16,9 \%)^{10}$.

A forma de prevenção da neoplasia de fígado consiste em controlar os fatores carcinogênicos mais relevantes por meio da prevençáo primária, sugerindo melhorias na cobertura vacinal da população contra os $\mathrm{HBV}$ e HCV, com uma assistência ampla e eficaz em todo território nacional, assim como medidas de maior segurança nas transfusôes de sangue e hemoderivados. O combate ao uso abusivo de álcool, esclarecendo a população sobre seus malefícios, também contribui significantemente na prevenção da doença. Todas essas medidas apresentam boa relação custo-efetividade e têm impacto direto na incidência da neoplasia de fígado e vias biliares.

Entretanto, ao ocorrer uma redução no impacto da mortalidade por câncer de fígado no Brasil, futuramente, haverá um direcionamento mais efetivo de açôes como o planejamento dos serviços, a identificação das unidades de referência, a regionalização do tratamento e a organização do fluxo de demanda de tratamento por Região ${ }^{26}$. Apesar de o Brasil contar com um sistema de saúde com cobertura universal, o desafio para os próximos anos será o desenvolvimento de um sistema capaz de responder à mudança dos riscos para a saúde e das necessidades de atendimento dos brasileiros a partir das alteraçóes demográficas e epidemiológicas pelas quais o país vem passando ${ }^{27}$.

De acordo com os resultados deste estudo, a projeçáo da mortalidade indica redução/estabilidade das taxas de mortalidade por esse câncer até 2030, principalmente nas Regióes Norte e Nordeste do Brasil. A perspectiva da reduçáo da mortalidade nas Regióes mais pobres do Brasil, de alguma forma, revela o enfrentamento que o país tem realizado para o controle dos fatores de risco para esse câncer.

O câncer de fígado e vias biliares, que está intimamente relacionado às Regióes menos desenvolvidas e com os menores níveis socioeconômicos, e que está relacionado às infecçôes virais e hábitos de vida e saúde, mostra uma faceta alentadora para a saúde pública do Brasil. O país tem mostrado redução considerável no número de mortes causadas por doenças infecciosas nas últimas seis décadas, e incluiu o acesso universal e gratuito à vacinação, o acesso ao tratamento e cuidados primários de saúde, além da instituição de políticas específicas para a vigilância e o controle dos fatores de risco, como a instituição do Plano de Açóes Estratégicas para o Enfrentamento das Doenças Crônicas não Transmissíveis, que tem, entre suas metas, a redução do consumo abusivo do álcool ${ }^{21,28}$.

Além disso, houve iniciativas focadas para a organização do diagnóstico e tratamento do câncer no país, a partir da elaboração da Política Nacional para a Prevenção e Controle do Câncer, que tem como objetivo a redução da mortalidade e da incapacidade causadas por essa doença e ainda a possibilidade de diminuir a incidência de alguns tipos de câncer, entre outras prerrogativas. Essas políticas, programas e açóes poderáo, em conjunto, ser a base para a explicação do sucesso total ou parcial para o controle dessa doença no presente e no futuro ${ }^{10,29}$.

Uma das limitaçóes deste estudo diz respeito ao registro nacional de óbitos no Brasil. É importante ressaltar que, no passado, foram observados problemas relacionados à confiabilidade e completude dos dados, particularmente nas Regiōes Norte e Nordeste do país. Os sub-registros de mortes em decorrência do câncer podem ser resultado da não exatidáo da definiçấo da causa de morte, e menores taxas de mortalidade pela doença podem ser decorrentes da classificação desses óbitos como causas mal definidas ou não especificadas. Ademais, o curto período de série temporal para realizar a análise de projeção de mortalidade também pode ser configurado como uma das limitaçóes do presente estudo.

Entretanto, embora sejam observadas algumas limitaçóes relacionadas às fontes de dados secundários provenientes de Sistemas de Informação em Saúde, algumas vantagens podem ser evidenciadas, como a ampla cobertura populacional, menor custo durante a coleta das informaçôes e o potencial dos dados para uso em pesquisas que reflitam na gestão de programas, políticas e serviços de saúde.

Todavia, desde a criaçáo dos sistemas de informação, o Estado brasileiro tem investido para o seu aperfeiçoamento, em particular na última década, obtendo em geral êxito expressivo $^{30}$. Além disso, a utilizaçáo de uma metodologia de correçáo do sub-registro minimiza o problema, tornando o estudo robusto e representativo da base populacional brasileira.

\section{CONCLUSÃO}

Em conclusão, neste estudo, a análise de dados mostra uma situação epidemiológica favorável relacionada ao câncer de fígado e vias biliares no Brasil, tendo em vista que as diferenças regionais na distribuição do câncer são mínimas, além das tendências temporais de redução e estabilidade que foram observadas nos últimos 15 anos e que continuarão a ser registradas nas próximas décadas. Os resultados das projeçôes apresentadas mostram que o 
perfil da mortalidade por esse câncer apresentará redução, principalmente para o sexo feminino, e que as políticas para a prevençáo e controle dos fatores de risco deverão ser continuadas e fortalecidas no país.

\section{CONTRIBUIÇÕES}

Isabelle Ribeiro Barbosa e Dyego Leandro Bezerra de Souza participaram do delineamento do estudo, análise dos dados, supervisão e redação final. Fábia Cheyenne Gomes de Morais Fernandes e Flávia Arichelle Cavalcante dos Santos participaram da coleta de dados, elaboraçáo dos bancos de dados e da redaçáo do manuscrito. Emelynne Gabrielly de Oliveira Santos e Nayre Beatriz Martiniano Medeiros participaram da redação e/ou revisão crítica. Todos os autores aprovaram a versáo final a ser publicada.

\section{DECLARAÇÃO DE CONFLITO DE INTERESSES}

Nada a declarar.

\section{FONTES DE FINANCIAMENTO}

Coordenação de Aperfeiçoamento de Pessoal de Nível Superior (Capes).

\section{REFERÊNCIAS}

1. Bray F, Ferlay J, Soerjomataram I, et al. Global cancer statistics 2018: GLOBOCAN estimates of incidence and mortality worldwide for 36 cancers in 185 countries. CA Cancer J Clin. 2018;68(6):394-424. doi: https://doi. org/10.3322/caac. 21492

2. Petrick JL, Braunlin $M$, Laversanne $M$, et al. International trends in liver cancer incidence, overall and by histologic subtype, 1978-2007. Int J Cancer, 2016;139(7):1534-45. doi: https://doi.org/10.1002/ijc.30211

3. Amorim TR, Merchán-Hamann E. Mortalidade por neoplasia maligna do fígado e vias biliares intrahepáticas no Brasil, 1980-2010. Cad Saúde Pública, 2013;29(7):1427-36. doi: http://dx.doi.org/10.1590/ S0102-311X2013000700016

4. Ferlay J, Colombet M, Soerjomataram I, et al. Cancer incidence and mortality patterns in Europe: estimates for 40 countries and 25 major cancers in 2018. Eur J Cancer. 2018;103:356-87. doi: http://dx.doi.org/10.1016/j. ejca.2018.07.005

5. Guimarães RM, Martins CA, Muzi CD, et al. Tendência para o câncer de fígado e vias biliares na Região Norte do Brasil. Rev Pan-Amaz Saúde. 2015;6(1):29-34. doi: http://dx.doi.org/10.5123/S2176-62232015000100004

6. Gomes MA, Priolli DG, Tralhão JG, et al. Carcinoma hepatocelular: epidemiologia, biologia, diagnóstico e terapias. Rev Assoc Med Bras. 2013;59(5):514-24. doi: http://dx.doi.org/10.1016/j.ramb.2013.03.005

7. Kumar V, Abbas AK, Aster JC. Robbins and Contran: pathologic basis of diseases. 9th ed. Philadelphia: Elsevier; 2015.

8. Bruix J, Reig M, Sherman M. Evidence-based diagnosis, staging, and treatment of patients with hepatocellular carcinoma. Gastroenterology. 2016;150(4):835-53. doi: http://dx.doi.org/10.1053/j.gastro.2015.12.041

9. Chedid MF, Kruel CR, Pinto MA, et al. Hepatocellular carcinoma: diagnosis and operative management. ABCD, Arq Bras Cir Dig. 2017;30(4):272-8. doi: http://dx.doi. org/10.1590/0102-6720201700040011

10. Melo APS, França EB, Malta DC, et al. Mortalidade por cirrose, câncer hepático e transtornos devidos ao uso de álcool: carga global de doenças no Brasil, 1990 e 2015. Rev Bras Epidemiol. 2017;20(Suppl 1):61-74. doi: http://dx.doi.org/10.1590/1980-5497201700050006

11. Wong MC, Jiang JY, Goggins WB, et al. International incidence and mortality trends of liver cancer: a global profile. Sci Rep. 2017;7:45846. doi: https://doi. org/10.1038/srep45846

12. Ministério da Saúde (BR), DATAUS, CoordenaçãoGeral de Disseminação de Informaçóes em Saúde. Mortalidade: redistribuiçáo por capítulos dos óbitos corrigidos pela pesquisa de busca ativa [Internet]. Brasília (DF): Ministério da Saúde; 2013 [acesso 2019 out. 20]. Disponível em: http://tabnet.datasus.gov.br/cgi/ deftohtm.exe?obitocorr/cnv/obitoredistr.def

13. Santos CA, Souza DLB. Melanoma mortality in Brazil: trends and projections (1998-2032). Cienc Saúde Coletiva. 2019;24(4):1551-61. doi: http://dx.doi. org/10.1590/1413-81232018244.13932017

14. Kim HJ, Fay MP, Feuer EJ, et al. Permutation tests for joinpoint regression with applications to cancer rates. Stat Med. 2000;19(3):335-51. doi: https://doi.org/10.1002/ (SICI) 1097-0258(20000215) 19:3<335::AIDSIM336>3.0.CO;2-Z

15. Boneca R, Payne P, Waterhouse JAH, editors. Cancer incidence in five continents. Genebra: Union Internationale Contre le Cancer; 1966. vol. I.

16. Møller B, Fekjaer H, Hakulinen T, et al. Prediction of cancer incidence in the Nordic countries: empirical comparison of different approaches. Stat Med. 2003;22(17):2751-66. doi: https://doi.org/10.1002/ sim. 1481

17. Sierra MS, Soerjomataram I, Antoni S, et al. Cancer patterns and trends in Central and South America. Cancer Epidemiol. 2016;44(Suppl 1):S23-S42. doi: https://doi.org/10.1016/j.canep.2016.07.013

18. Souto FJD. Distribution of hepatitis B infection in Brazil: the epidemiological situation at the beginning of the $21^{\text {st }}$ century. Rev Soc Bras Med Trop. 2016;49(1):11-23. doi: http://dx.doi.org/10.1590/0037-8682-0176-2015 
19. Silva RSU, Morais IO, Gonçalves DM, et al. Prevalência da infecção pelo vírus da hepatite $\mathrm{B}$ em um município do interior do estado do Acre, Amazônia Ocidental, Brasil. Rev Pan-Amaz Saúde. 2017;8(3):19-26. doi: http:// dx.doi.org/10.5123/s2176-62232017000300003

20. Secretaria de Estado da Saúde de Sáo Paulo, Coordenadoria de Controle de Doenças, Centro de Vigilância Epidemiológica "Prof. Alexandre Vranjac". Estudo de prevalência de base populacional das infecçóes pelos vírus das hepatites $\mathrm{A}, \mathrm{B}$ e $\mathrm{C}$ nas capitais do Brasil. Bol Epidemiol Paulista. 2007;4(44):23-24.

21. Barreto ML, Teixeira MG, Bastos FI, et al. Sucessos e fracassos no controle de doenças infecciosas no Brasil: o contexto social e ambiental, políticas, intervençôes e necessidades de pesquisa. Lancet [Internet]. 2011 maio [acesso 2019 out. 20]:47-60. (Séries Saúde no Brasil; 3). Disponível em: https://www.thelancet.com/pb/assets/ raw/Lancet//pdfs/brazil/brazilpor3.pdf

22. World Health Organization. Hepatitis C [Internet]. Geneva: WHO; 2019 July [cited 2019 Oct. 20]. Available from: https://www.who.int/en/news-room/fact-sheets/ detail/hepatitis-c

23. Nunes HM, Soares MCP, Sarmento VP, et al. Soroprevalência da infecção pelos vírus das hepatites A, B, C, D e E em município da região Oeste do Estado do Pará, Brasil. Rev Pan-Amaz Saúde. 2016;7(1):55-67. doi: http://dx.doi.org/10.5123/S2176-62232016000100007

24. Ministério da Saúde (BR), Secretaria de Vigilância em Saúde, Coordenação-Geral de Desenvolvimento da Epidemiologia em Serviços. Guia de vigilância em saúde [Internet]. 3. ed. Brasília, DF: Ministério da Saúde; 2019 [acesso 2019 out. 20]. Disponível em: http://portalarquivos2.saude.gov.br/images/pdf/2019/ junho/25/guia-vigilancia-saude-volume-unico-3ed.pdf

25. Garcia LP, Freitas LRS. Consumo abusivo de álcool no Brasil: resultados da Pesquisa Nacional de Saúde 2013. Epidemiol Serv Saúde. 2015;24(2): 227-237. doi: http:// dx.doi.org/10.5123/S1679-49742015000200005

26. Knaul FM, Alleyne G, Piot P, et al. Health system strengthening and cancer: a diagonal response to the challenge of chronicity. In: Knaul FM, Gralow JR, Atun R, et al., editors. Closing the cancer divide: an equity imperative. Cambridge: Harvard Global Equity Initiative; 2012. p. 79-95.

27. Barbosa IR, Souza DLB, Bernal MM, et al. Cancer mortality in Brazil: temporal trends and predictions for the year 2030. Medicine (Baltimore). 2015;94(16):e746. doi: http://dx.doi.org/10.1097/MD.0000000000000746

28. Ministério da Saúde (BR), Secretaria de Vigilância em Saúde, Departamento de Análise de Situação de Saúde. Plano de açóes estratégicas para o enfrentamento das doenças crônicas não transmissíveis (DCNT) no Brasil 2011-2022. Brasília (DF): Ministério da Saúde; 2011. (Série B. Textos Básicos de Saúde).
29. Ministério da Saúde (BR). Portaria no 874, de 16 de maio de 2013. Institui a Política Nacional para a Prevenção e Controle do Câncer na Rede de Atençáo à Saúde das Pessoas com Doenças Crônicas no âmbito do Sistema Único de Saúde (SUS). Diário Oficial da União, Brasília, DF; 2013 maio 17. Seção I, p. 129.

30. Frias PG, Szwarcwald CL, Morais Neto OL, et al. Utilização das informaçóes vitais para a estimação de indicadores de mortalidade no Brasil: da busca ativa de eventos ao desenvolvimento de métodos. Cad Saúde Pública. 2017;33(3):e00206015. doi: http://dx.doi. org/10.1590/0102-311x00206015

Recebido em 31/8/2019 Aprovado em 11/11/2019 